A new technique to measure small eye movements is reported. The precise recording of human eye movements is necessary for research on visual fatigue induced by visual display units. ${ }^{1}$ So far all methods used have disadvantages: especially those which are sensitive or are rather painful. ${ }^{2,3}$ Our method is based on a transformation of mechanical vibrations into magnetic flux variations. In order to do this a small magnet is embedded in a closefitting soft contact lens. The magnetic flux variations caused by eyeball movements during fixation are measured by means of a SOUID magnetometer. The recordings show the typical fixation pattern of a human eye. This pattern is composed of three kinds of movements: saccades, drift and microtremor. The last-mentioned type of movements are displacements in the order of $2 \mu \mathrm{m}$. It is possible to distinguish between movements which are perpendicular to each other.

\title{
Measuring miniature eye movements by means of a SOUID magnetometer
}

\author{
M.J. Peters, Z. Dunajski, T.E.M. Meijzssen, E.W. Breukink and J.J. Wevers-Henke
}

Key words: superconducting devices, squid, magnetometer; eye movement

\section{Set-up and measuring procedure}

The measurements are done in a magnetically unshielded room. The SQUID magnetometer consists of a SQUID sensor, connected to a symmetrical second-order gradiometer with a baseline of $6 \mathrm{~cm}$ and a diameter of $3 \mathrm{~cm} .{ }^{4}$ This assembly is immersed in a cryostat especially designed for biomagnetic measurements. The distance between pick-up coil and the outer wall of the cryostat is only $1 \mathrm{~cm}$. The SQUID is coupled to SQUID electronics (SHE 330x). The output voltage of the electronics is linearly proportional to the variations of the magnetic flux in the flux transformer. The output voltage is filtered by a notch filter at $50 \mathrm{~Hz}$ and a $48 \mathrm{~dB} /$ oct low-pass Butterworth filter. The magnetic sample is made of Samarium cobalt $\mathrm{V}$ and the dimensions are $1.2 \times 0.8 \times 0.15 \mathrm{~mm}$. The magnetic dipole moment was found to be $m=0.8 \times 10^{-4} \mathrm{Am}^{2}$. This magnet is mounted by means of a very small incision in a soft contact lens. The lens is 'sucked' onto the cornea by choosing an inner curvature of the lens smaller than that of the cornea. In order not to interfere with normal vision the magnet is placed a little excentrically. In this way the subject does not experience any irritation at all. The magnetic dipole moment points perpendicularly to the lens. For practical reasons we have chosen two different measuring positions which are sketched in Fig. 1. In case a) the subject is in supine position on a wooden bed, in case b) the subject is turned on his side. The cryostat is vertical, so the loops of the flux transformer are horizontal. In order to avoid head movement artifacts, which are mainly evoked by pumping of the heart, the head is fixed to the bed with two rubber strips. As a check, an electrocardiogram of the subject is recorded simultaneously.

\section{Theory}

The small magnet is considered as a magnetic dipole with magnetic dipole moment $\mathrm{m}$. The magnetic induction caused

MJP, TEMM and JJWH are from Twente University of Technology, Enschede. The Netherlands, ZD is from the Institut for Biophysics and Biocybernetics, Warsaw, Poland, and EWB is an ophtalmologist, Enschede, The Netherlands. Paper received 3 February 1982. by a dipole is given by:

$$
\mathbf{B}(\mathbf{r})=\frac{\mu_{0} m}{4 \pi r^{3}}\left(2 \mathbf{e}_{\mathrm{r}} \cos \theta+\mathbf{e}_{\theta} \sin \theta\right)
$$

where $r$ is the radius vector drawn from the dipole to the point of observation; $\theta$ is the angle from $m$ to $r$; $\mathbf{e}_{\mathbf{r}}$ is the unit vector in the direction of $\mathbf{r} ; \mathbf{e}_{\theta}$ is the unit vector in the plane of $\mathbf{m}$ and $\mathbf{r}$ in the direction corresponding to increasing $\theta$.

The total flux through all loops of the flux transformer is:

$$
\begin{aligned}
& \phi=\phi_{1}-2 \phi_{2}+\phi_{3}, \text { with } \phi_{\mathrm{i}}=\iint \mathbf{B}_{\mathrm{i}} \cdot \mathrm{d} \mathbf{S}_{\mathrm{i}}=\text { flux } \\
& \text { through loop } i
\end{aligned}
$$

A cartesian coordinate system is chosen so that the origin is in the centre of the eyeball. The eyeball is considered as a sphere with a radius $R_{0}=1.55 \times 10^{-2} \mathrm{~m}$. The magnet is situated on and pointing normal to the surface of the sphere. We take the $x$-axis perpendicular to the loops of the flux transformer and the centres of the loops are in the plane $y=0$, (see Fig. 2). The coordinates of the centre of loop i are $\left(M_{\mathrm{x}}, 0, M_{\mathrm{z}}\right)$. An arbitrary point within the loop is given by $\left(M_{\mathrm{x}}, \rho \sin \phi, M_{\mathrm{z}}+\rho \cos \phi\right)$.

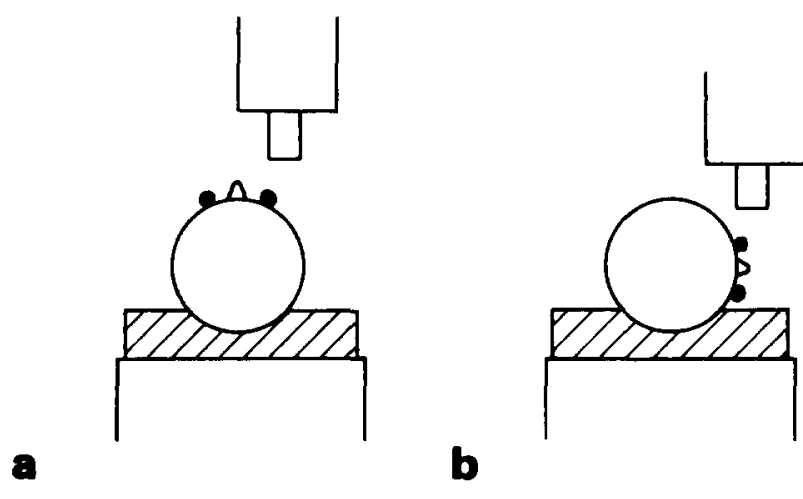

Fig. 1 Schematic representation of the two chosen measuring positions 


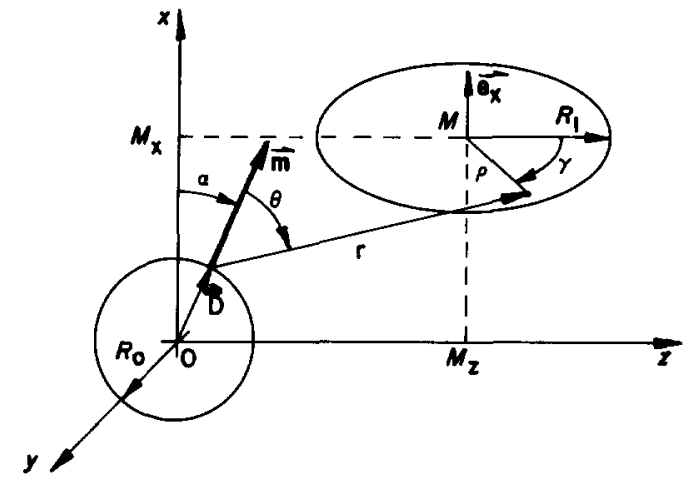

Fig. 2 The coordinate system used to compute the magnetic flux through a single loop caused by a magnetic dipole which can rotate over the surface of a sphere, representing the eyeball (measuring position as given in Fig. 1a)

In situation a) (the subject in supine position, see Fig. 1) the magnet is pointing initially along the $x$-axis. The variable angle between the direction of the rotating dipole and the $x$-axis is termed $\alpha$. The position and the direction of the dipole is given by the vector $D$. This means that in the case where the dipole is rotating in the $x z$-plane $\mathrm{D}=\left(R_{0} \cos \alpha\right.$, $0, R_{0} \sin \alpha$ ) and where the dipole is rotating in the $x y$-plane $\mathrm{D}=\left(R_{0} \cos \alpha, R_{0} \sin \alpha, 0\right)$.

In situation $b$ ) (the subject turned on his side) the starting position of the magnet is along the $z$-axis. The angle between the direction of the rotating dipole and the $z$-axis is termed $\alpha$. The position and direction of the dipole is given by the vector $D$. In the case where the dipole is rotating in the $x z$-plane $\mathrm{D}=\left(R_{0} \sin \alpha, 0, R_{0} \cos \alpha\right)$ and where the dipole is rotating in the $y z$-plane $\mathrm{D}=\left(0, R_{0} \sin \alpha, R_{0} \cos \alpha\right)$.

The flux through loop i, with radius $R_{\mathrm{i}}$ is:

$$
\phi_{\mathrm{i}}=\int_{0}^{R_{\mathrm{i}}} \int_{0}^{2 \pi} \mathbf{B} \cdot \mathbf{e}_{\mathrm{x}} \rho \mathrm{d} \rho \mathrm{d} \phi
$$

We insert B from (1).

$$
e_{\theta}=\frac{e_{r} x\left(e_{r} x e_{D}\right)}{\mid e_{T} x\left(e_{r} x e_{D}\right)}
$$

where $\mathbf{e}_{\mathbf{D}}=\frac{\mathbf{D}}{|\mathrm{D}|}$

We rewrite the expression for $\mathrm{e}_{\theta}$ with the help of the vector identity $\mathbf{a x}(\mathbf{b x c})=\mathbf{b}(\mathbf{a} \cdot \mathbf{c})-\mathbf{c}(\mathbf{a} \cdot \mathbf{b})$, which gives:

$$
\mathbf{e}_{\theta}=\frac{\mathbf{e}_{\mathrm{r}} \cos \theta-\mathbf{e}_{\mathrm{D}}}{\sin \theta}
$$

For both experimental situations a) and b) integral (3) can be computed numerically using Simpson's rule. Inserting $R_{0}=1.55 \times 10^{-2} \mathrm{~m} ; m=1 \mathrm{Am}^{2} ;$ for all loops $R=1.5 \times 10^{-2} \mathrm{~m}$ and a baseline length $H=6 \times 10^{-2} \mathrm{~m}$, the total flux through the flux transformer is calculated. The results are given in the Figs 3 and 4 , where $\phi$ is given as a function of $M_{z}$ for different values of $M_{\mathbf{x}}$.

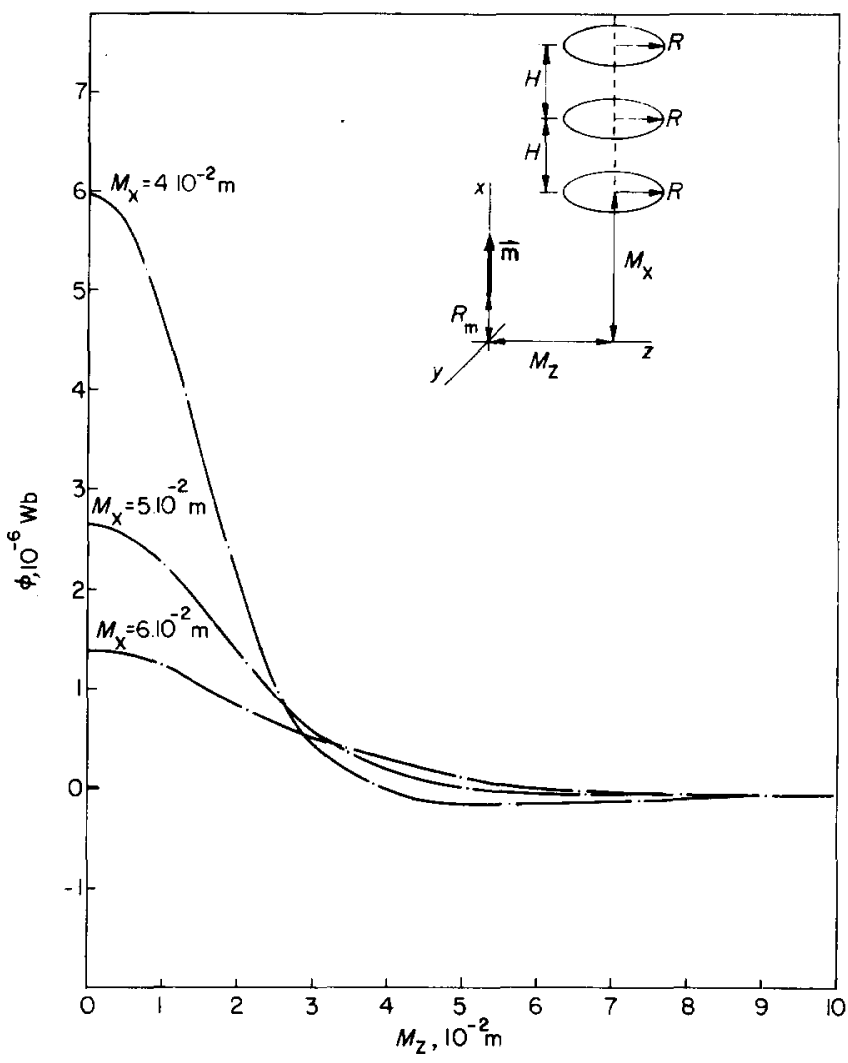

Fig. 3 The flux through the given flux transformer caused by a magnetic dipole pointing parallel to the axis of the flux transformer, with $|\mathrm{m}|=1 \mathrm{Am}^{2}$ (measuring position as given in Fig. 1a)

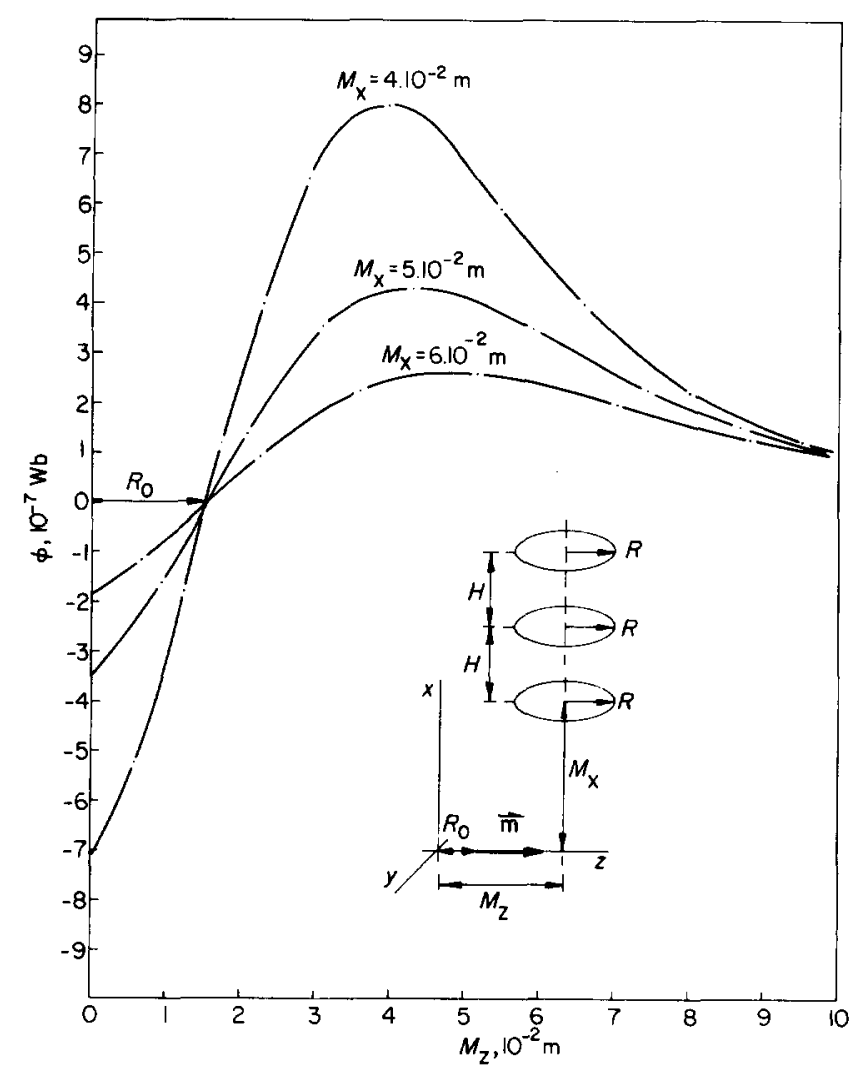

Fig. 4 The flux through the given flux transformer caused by a magnetic dipole pointing perpendicular to the axis of the flux transormer, with $|\mathrm{m}|=1 \mathrm{Am}^{2}$ (measuring position as given in Fig. 1b)

The total flux change as a result of a small rotation of the dipole through an angle $\Delta \alpha$ (starting at $\alpha=0$ ) can be 
approximated by means of the first two terms of a Taylor's series expansion:

$$
\Delta \phi=\left(\frac{\partial \phi}{\partial \alpha}\right)_{\alpha=0} \Delta \alpha+\left(\frac{\partial^{2} \phi}{\partial \alpha^{2}}\right)_{\alpha=0} \frac{(\Delta \alpha)^{2}}{2}
$$

We took $\Delta \alpha=0.15 \mathrm{~m} \mathrm{rad}$, which represents a displacement of $2 \mu \mathrm{m}$. The total flux change through the flux transformer is calculated (taking $m=1 \mathrm{Am}^{2}$ ) for rotations in two directions which are perpendicular to each other for both types of experimental situations $a$ ) and $b$ ).

The results are given in Figs 5, 6, 7 and 8. Looking at these results we can draw the following conclusions:

1. In situation a) the sensitivity is maximal for $M_{\mathrm{z}} \approx 2 \mathrm{~cm}$ (slightly dependent on $M_{\mathrm{x}}$ ) when the rotation takes place in the $x z$-plane and for $M_{\mathrm{z}}=0$ when the rotation takes place in the $x y$-plane. In situation b) maximal sensitivity is found for $M_{\mathrm{z}}=2.5 \mathrm{~cm}$ when the rotation is in the $x z$-plane and for $M_{z}=3.5 \mathrm{~cm}$ when the rotation is in the $y z$-plane.

2. In situation a) and $M_{\mathrm{z}}=2.0 \mathrm{~cm}$ the sensitivity for rotations in the $x z$-plane is 9000 times as large as for rotations in the $x y$-plane. In situation b) and $M_{\mathrm{z}}=$ $2.5 \mathrm{~cm}$ the sensitivity is 15000 times larger for rotations in the $x z$-plane than for rotations in the $y z$-plane.

These results show the possibility to distinguish the direction of the rotation. The two types of rotations may be recorded simultaneously with two magnetometers. The sensitivity is high in the case where the first order term of our series expansion is high with respect to the second order

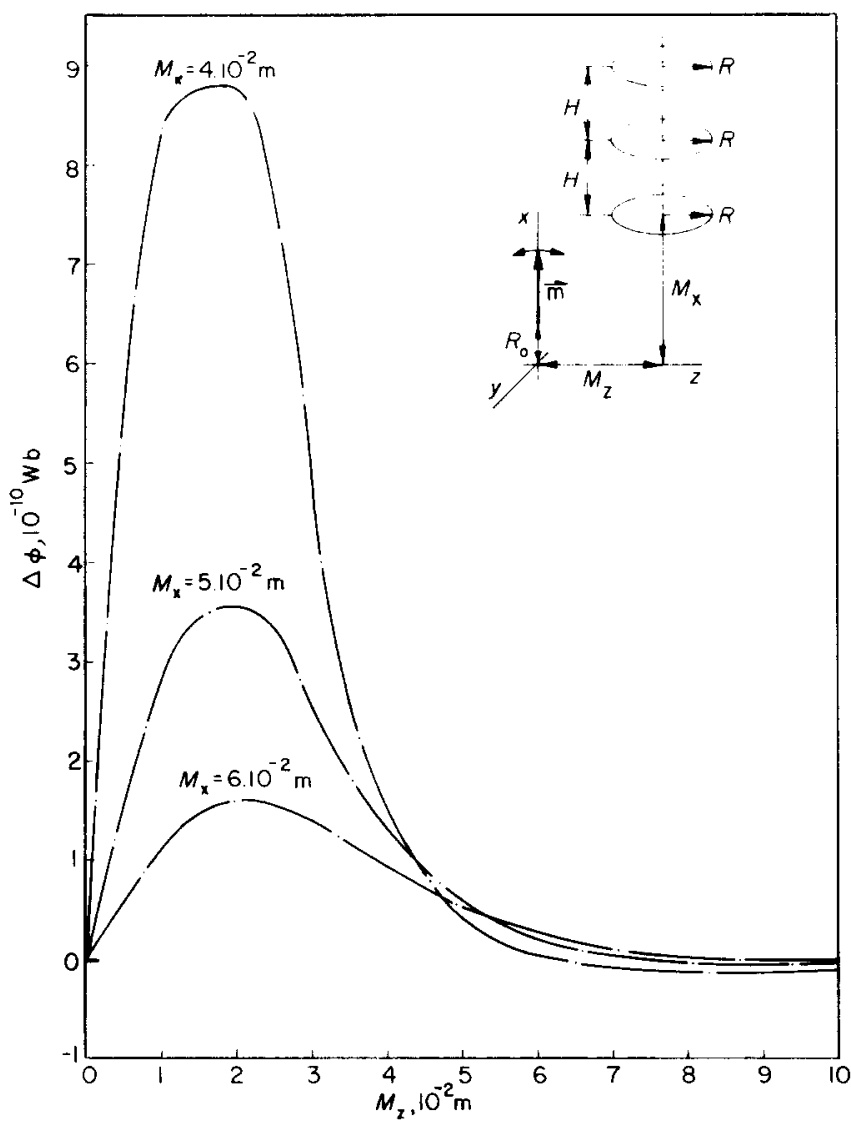

Fig. 5 The flux change through the given flux transformer caused by a rotation of the given dipole of $0.15 \mathrm{mrad}$ in the $x z$-plane. Im $\mid=1 \mathrm{Am}^{2}$

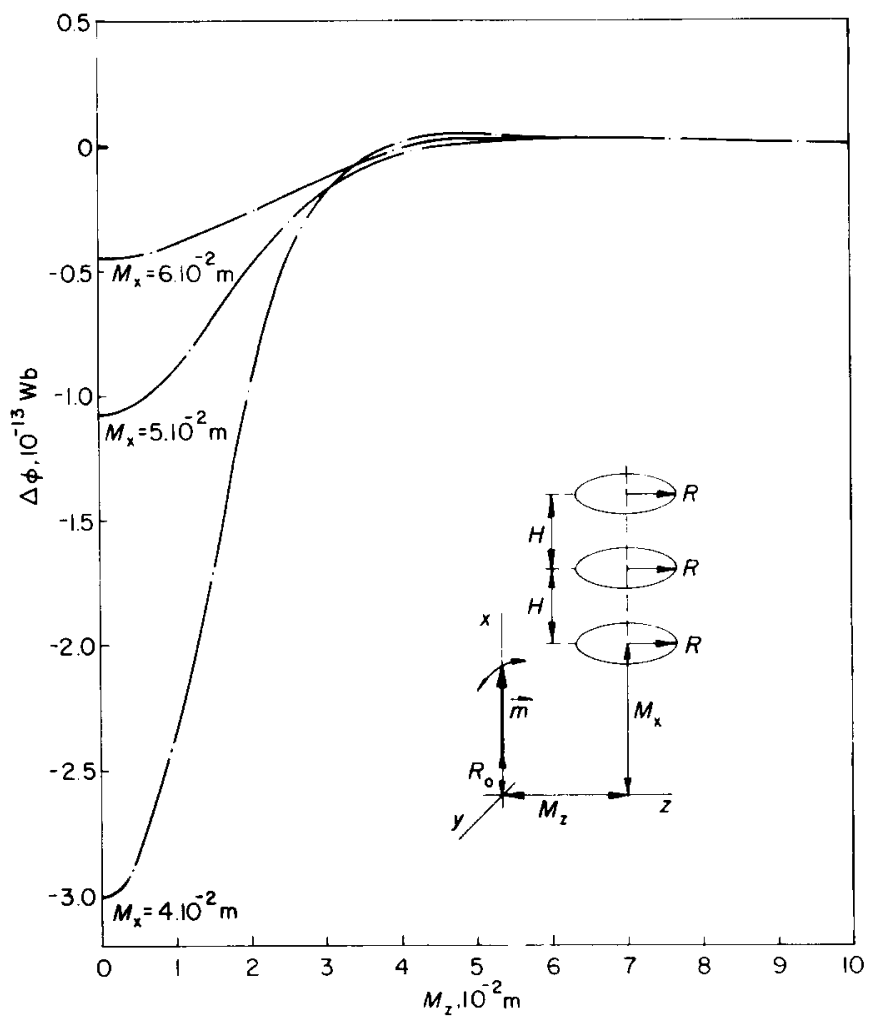

Fig. 6 The flux change through the given flux transformer caused by a rotation of the given dipole of $0.15 \mathrm{mrad}$ in the $x y$-plane. $|\mathrm{m}|=1 \mathrm{Am}^{2}$

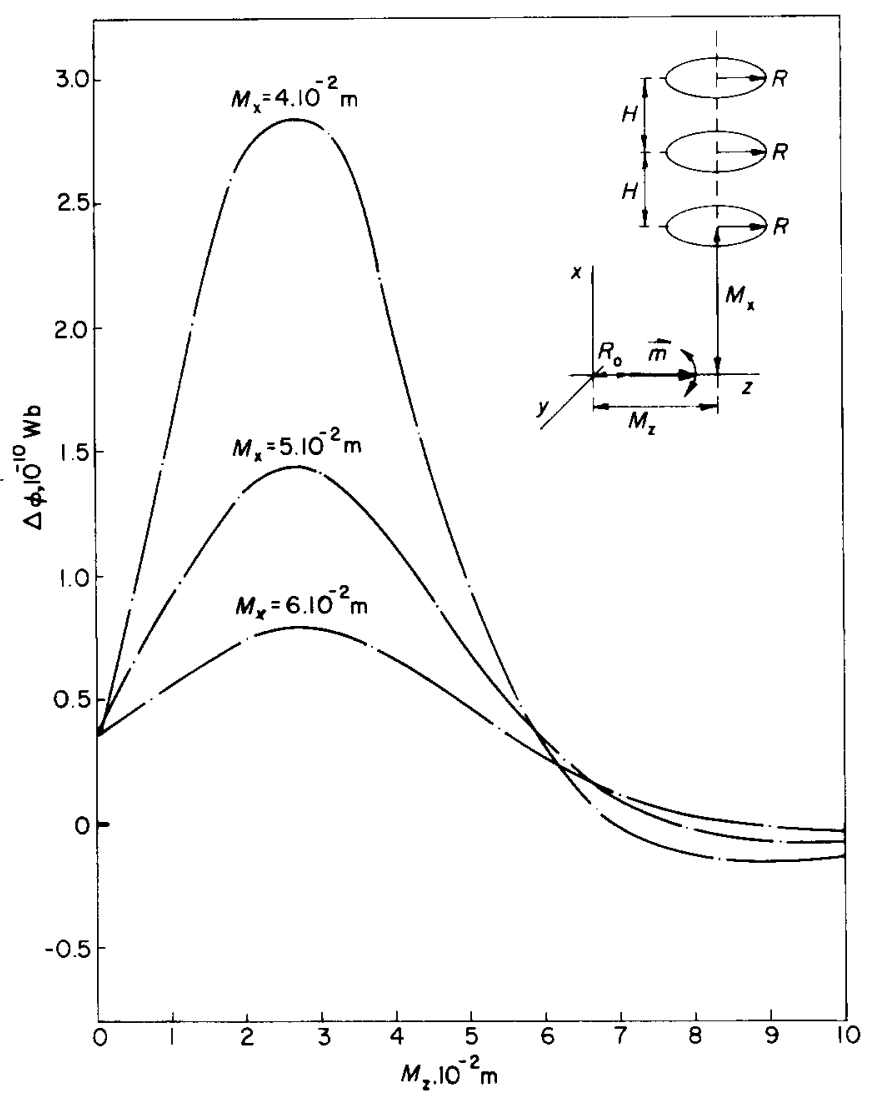

Fig. 7 The flux change through the given flux transformer caused by a rotation of the given dipole of $0.15 \mathrm{mrad}$ in the $x z$-plane. $|\mathrm{m}|=1 \mathrm{Am}^{2}$

term. This means that in this case the flux change, ie the output voltage of our magnetometer, is practically linearly proportional to $\Delta \alpha$. 


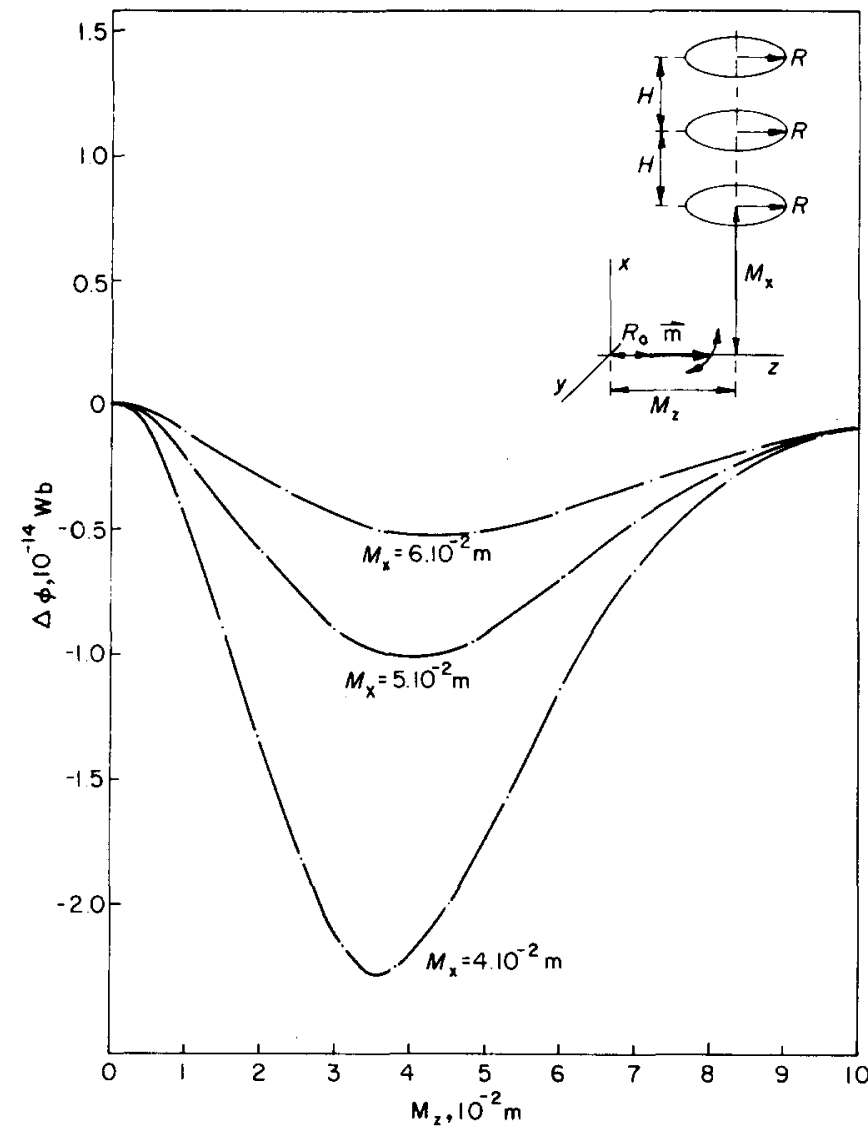

Fig. 8 The flux change through the given flux transformer caused by a rotation of the given dipole of $0.15 \mathrm{mrad}$ in the $y z$-plane. $|\mathrm{m}|=1 \mathrm{Am}^{2}$

\section{Experimental results and conclusions}

The output voltage for rotations in the $x z$-plane was found to be $8 \mathrm{~V}$ for a known rotation of the eyes of $1^{\circ}$. This means that a rotation of $0.15 \mathrm{mrad}$ will give an output of about $70 \mathrm{mV}$. The actual noise, ie the output of the magnetometer without the magnet fixed on the eye, was about $10 \mathrm{mV}$.

In Fig. 9 a typical pattern is shown during fixation on a point. This pattern is composed of three kinds of movements: saccades, drift and microtremor. Saccades are small, very fast changes in eye position, which occur one to three times each second. In the intervals between saccades the eye slowly drifts back and forth. Saccades and drift are superimposed upon a high frequency tremor with frequencies up to $100 \mathrm{~Hz}$ or more. During the recording no influence of the pumping of the heart was noted. The analogue filter had its $3 \mathrm{~dB}$ point at $120 \mathrm{~Hz}$. The actual distances between the centre of the eyeball and the centres of the loops of the

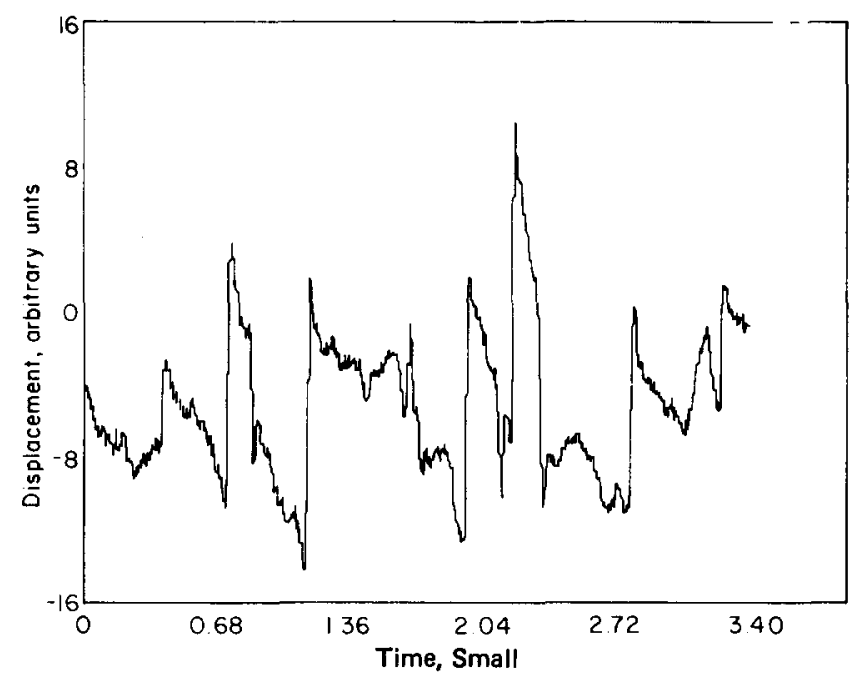

Fig. 9 Eye movements during fixation of the eye onto which the contact lens with the magnet is fixed

flux transformer are difficult to establish accurately.

We found a difference in sensitivity of a factor 100 for rotations in different directions. These results were found by asking the subject of the experiment to scan the edges of a shown square with his eyes. When the subject was asked to move his eyes as far and rapidly as possible and immediately after that again to focus on the fixation point the output voltage regained its initial value. This gives the impression that the lens is following the eye movements quite well.

Keeping track of a circle or another figure with the eyes gives signals with such high amplitudes that these displacements may be seen by means of a flux gate magnetometer.

We conclude that the SQUID magnetometer method has proved to be a simple, sensitive and painless method for measuring very small rotations of the human eye.

The authors would like to thank Professor Dr L.C. van der Marel, Dr H.J.M. Gerrits and Professor D. Bosman for their helpful discussions and J.A. Ulfman for his technical assistance.

\section{References}

1 Umbach, F.W., Kalsbeek, J.W.H., Bosman, D. Proc of the first European display research conf (1981) Munich

2 Yarbus, A.L. Eye movements and vision, Plenum Press New York (1967)

3 Collewijn, H., V.d. Mark, F., Jansen, T.C. Vision Res. 15 Pergamon Press (1975) 447

4 Peters, M.J. thesis (1981); Twente University of Technology, The Netherlands 